\title{
Under the influence: examination of prevalence and correlates of alcohol and marijuana consumption in relation to youth driving and passenger behaviours in Canada. A cross-sectional study
}

\author{
Leia M. Minaker PhD, Aaron Bonham MSc, Tara Elton-Marshall PhD, Cesar Leos-Toro BSc, \\ T. Cameron Wild PhD, David Hammond PhD
}

Abstract

Background: Consequences of alcohol- and drug-impaired driving affect youth disproportionately. We describe individual- and arealevel characteristics associated with risky driving and passenger behaviours among grade 9-12 students in Canada.

Methods: The 2014-2015 Canadian Student Tobacco, Alcohol and Drug Survey was administered to 24650 students in provincially generalizable samples. Dichotomous outcomes included ever and last-30-day driving after drinking alcohol, ever and last-30-day driving after using marijuana, and ever and last-30-day reporting of being a passenger with a driver who had been drinking or using marijuana.

Results: A total of $9.1 \%$ (99\% confidence interval 7.9-10.3) of grade 11-12 students reported ever driving after drinking, and $9.4 \%$ (99\% confidence interval 8.3-10.4) reported ever driving after using marijuana. Almost half (48\%) of grade 11-12 students reported ever participating in any risky driving or passenger behaviour. Over one-third (35\%) of grade 9-12 students reported ever riding with a driver who had been drinking, and $20 \%$ reported ever riding with a driver who had been using marijuana. Logistic regression models showed that boys had higher odds of risky driving behaviours relative to girls, whereas girls had higher odds of risky passenger behaviours relative to boys. Students from rural schools had higher odds of drinking and driving and of riding with a driver who had drunk relative to students from urban schools. There were significant differences in risky driving and passenger behaviours by province.

Interpretation: A substantial number of Canadian youth reported risky driving and passenger behaviours, which varied by individual and area-level characteristics. Federal marijuana policy should aim to reduce the prevalence of drug-impaired driving. Additional provincial policies to prevent impaired driving are needed.

U nintentional injuries are the leading cause of death among Canadians aged 1-24 years, being responsible for $35 \%$ of deaths in this age group. ${ }^{1-3}$ Traffic collisions account for a high proportion of accident-related deaths and injuries and place a substantial burden on the health care system. ${ }^{4}$ Young drivers are at higher risk for collision-related mortality than drivers in other age groups, ${ }^{5}$ and $39 \%$ of car-crash deaths among 16 - to 19 -year-olds were related to alcohol in $2012 .^{6}$

Alcohol is the most common intoxicant in Canada, ${ }^{7}$ and the link between alcohol-impaired driving and high collision rates is well recognized. ${ }^{8,9}$ Less frequently studied are adolescents' decisions to ride with impaired drivers. In 2008, $15 \%$ of Canadians aged 15-17 years rode with an alcohol-impaired driver, and $19 \%$ rode with a cannabis-impaired driver. ${ }^{10}$ In 20092010, about 20\% of grade 6-10 Canadian students rode with a driver who had consumed alcohol, cannabis or other drugs in the previous 30 days. ${ }^{11}$ Younger age (9-15 yr), male sex, heavy drinking, lower socioeconomic status and rural residence are associated with riding with an impaired driver. ${ }^{10-13}$

Cannabis is second most commonly used intoxicant in Canada: ${ }^{14} 17 \%$ of Canadian youth reported cannabis use in 2014-2015. ${ }^{7}$ Cannabis-impaired driving has recently come to the fore given the Canadian government's plan to legalize the possession and sale of marijuana to adults. Proposed legislation to reduce drug-impaired driving was tabled in October 2016 in the Canadian Senate,,$^{15}$ and, in the absence of current federal law, Canadian provinces have begun to introduce leg-

\section{Competing interests: None declared.}

This article has been peer reviewed.

Correspondence to: Leia Minaker, Iminaker@uwaterloo.ca

CMAJ Open 2017. DOI:10.9778/cmajo.20160168 
islation to address drug-impaired driving and create heavier sanctions. ${ }^{16}$ Debate exists regarding the extent to which driving under the influence of cannabis causes motor vehicle fatalities. ${ }^{17-21}$ In Ontario in 2015, 12\% of grade 7-12 students rode with a driver who had used drugs, including cannabis, at least once in the previous year. ${ }^{22}$

In light of the impending legalization of cannabis in Canada, we aimed to determine what individual and area-level characteristics are associated with risky driving and passenger behaviours among grade 9-12 students in Canada.

\section{Methods}

\section{Design}

The Canadian Student Tobacco, Alcohol and Drugs Survey is a biennial, provincially generalizable, paper-and-pencil, schoolbased survey administered to students across Canada. ${ }^{7}$ The survey uses a stratified single-stage cluster design; strata are based on the rate of cigarette smoking in the health region and type of school. In each province, 2 or 3 smoking rate strata and 2 schoollevel strata (elementary and high school) are defined. Schools are randomly selected within each stratum to ensure a generalizable sample within each province. The 2014-2015 survey wave did not include a generalizable sample of students in New Brunswick owing to a low response rate. National estimates include data from the 3 New Brunswick schools that participated.

\section{Setting}

The survey was conducted in private, public and Catholic schools attended by grade 6-12 students (6 to secondary $\mathrm{V}$ in Quebec) in all 10 provinces. Data were collected between October 2014 and May 2015.

\section{Participants}

Within each participating school, all students in eligible grades (6-12) were invited to participate in the survey. Schools and school boards determined permission protocols. About $66 \%$ of students participated with active informationpassive permission, and 34\% participated with active parental permission. Sample size was based on the ability of the sample to provide generalizable estimates at the provincial level.

\section{Measures}

Dichotomous outcomes from the survey included ever and previous-30-day experiences of 1) driving within 1 hour of drinking alcohol, 2) driving within 2 hours of using marijuana, 3) being a passenger in a vehicle driven by someone who had consumed alcohol within the previous hour and 4) being a passenger in a vehicle driven by someone who had used marijuana in the previous 2 hours. The first 2 outcomes were derived from survey responses to the question "Have you driven a vehicle (e.g., car, snowmobile, motor boat or all-terrain vehicle) within an hour of drinking one or more drinks of alcohol, or within 2 hours of using marijuana?" Response options included "No, never," "Yes, in the last 30 days" and "Yes, more than 30 days ago." Outcomes 3 and 4 were derived from survey responses to the question "Have you ever been a passenger in a vehicle (e.g., car, snowmobile, motor boat, or all-terrain vehicle) a) driven by someone who had 1 or more drinks of alcohol in the last hour? and b) driven by someone who had been using marijuana in the last 2 hours?," with the same response options as above. For all 4 outcomes of interest, we created dichotomous variables to assess "Ever" (those responding with either "yes" option) and "Last 30 days" (those responding "Yes, in the last 30 days") driving and passenger behaviours.

Independent variables included respondents' sex (female, male), grade (9-12 [age 13-18 yr in Canada]), province of residence (with Ontario as the reference province, given that it is the most populous province in Canada), binge drinking behaviours among students who reported consuming alcohol (drinking but never binge drinking [drinking at least 5 drinks on 1 occasion] and ever binge drinking) and race/ethnicity (white, black, Asian, Aboriginal, Latin American or other).

We examined 2 area-level independent variables: schoolregion socioeconomic status and rural versus urban school location. The median family income of the school's forward sortation area (first 3 digits of the postal code) from the 2011 census was dichotomized at the provincial median and was treated as a dichotomous variable (high and low socioeconomic status). Urban and rural categories were based on Statistics Canada's Statistical Area Classification system and were derived from school postal codes. Urban areas were considered census metropolitan areas (total population of at least 100 000, of whom 50000 live in the core) or census agglomerations (core population of at least 10 000), which are areas consisting of at least 1 neighbouring municipality situated around a core. ${ }^{23}$ Rural areas were considered noncensus metropolitan areas or census agglomerations. We adopted these definitions of socioeconomic status and urban versus rural locale since they are in the survey's public use microdata file (Vicki Rynard, Propel Centre for Population Health Impact, Waterloo, Ont.: personal communication, 2015).

\section{Statistical analysis}

In Canada, adolescents can operate a motor vehicle on their own between 16 and 17 years of age (Appendix 1, available at www.cmajopen.ca/content/5/2/E386/suppl/DC1), which generally corresponds to the age of grade 11 students. Therefore, in the absence of drivers' licence data, we restricted analyses to grade 11-12 students for driving outcomes and included grade 9-12 students in analyses examining passenger outcomes.

We used survey weights to adjust for sample selection, nonresponse (school, grade and student levels) and calibration of the sample to the grade and sex distribution of the target population. We used bootstrap weights for all analyses to account for survey design effects on variance estimates. One important effect of using the bootstrap weights is the adjustment of estimate and model variances for clustering within schools. Consequently, similar adjustment of models by entering schools as random effects is not necessary.

We used descriptive statistics to show the weighted prevalence of driving and passenger outcomes of interest according to the independent variables listed above. We produced weighted logistic regression models to examine independent variables associated with ever and last-30-day driving after 


\section{OPEN}

Research

drinking, driving after using marijuana, riding with a driver who had drunk and riding with a driver who had used marijuana. Covariates for each model included respondents' sex, grade, ethnicity, drinking behaviours, school-level socioeconomic status and school-level urban versus rural locale. We conducted logistic regression analyses with bootstrap weight adjustment for clustering within schools using PROC SURVEYLOGISTIC in SAS version 9.4 (SAS Institute).

\section{Ethics approval}

The study received ethics approval from the Health Canada Research Ethics Board, the Office of Research Ethics at the University of Waterloo and ethics review boards located at the institutions and school boards in each province.

\section{Results}

A total of 177 school boards (68\% of those approached), 336 schools (47\% of those approached) and 24650 grade 9-12 students (66\% recruitment rate) participated in the Canadian Student Tobacco, Alcohol and Drugs Survey. Although reasons for nonparticipation varied, school boards reported participation in other research as their primary reason not to participate in the survey, and schools reported being "too busy" to participate as their primary reason for nonresponse. Student nonparticipation is primarily influenced by the parental permission protocol used at the school. There is a higher nonparticipation rate in schools requiring active permission methods in comparison to active information-passive permission methods. For each outcome variable, less than $5 \%$ of data were missing (range 2.6\% [driving after drinking] to $3.6 \%$ [driving after cannabis use]).

Table 1 presents sample characteristics. Overall, 9.1\% of Canadian grade 11-12 students reported ever driving within an hour of drinking at least 1 drink (Table 2). A similar proportion $(9.4 \%)$ reported ever driving within 2 hours of using marijuana (Table 3 ). A total of $34.6 \%$ of grade 9-12 students reported ever being passengers of a driver who had had at least 1 drink within the previous hour (Table 4), and 19.8\% reported ever riding with a driver who had used marijuana within the previous 2 hours (Table 5). The prevalence of ever driving after drinking ranged from a low of $6.3 \%$ in Ontario to a high of $18.5 \%$ in Saskatchewan, and the prevalence of ever driving after using marijuana ranged from a low of $6.9 \%$ in Quebec to $20.0 \%$ in Saskatchewan.

In the overall sample, $48.1 \%$ of grade $11-12$ students (representing 351900 students [weighted Canadian population estimate]) reported ever engaging in any risky driving or passenger behaviours: $25.5 \%$ (representing 186400 students) reported engaging in 1 behaviour, and 22.6\% (representing 165600 students) reported engaging in more than 1 behaviour.

Girls had lower odds than boys of ever driving after drinking (odds ratio [OR] $0.477,99 \%$ confidence interval [CI] 0.290-0.786) or after using marijuana (last 30 days OR 0.448, 99\% CI 0.240-0.834; ever OR 0.517, 99\% CI 0.333-0.805). Conversely, compared to boys, girls had higher odds of ever riding with a driver who had drunk in the previous hour (OR $1.337,99 \%$ CI 1.159-1.543).
Table 1: Characteristics of grade 9-12 students who participated in the 2014-2015 Canadian Student Tobacco, Alcohol and Drugs Survey

\begin{tabular}{|c|c|c|}
\hline Characteristic & Sample size & $\begin{array}{l}\text { Weighted \%* } \\
\quad(99 \% \mathrm{Cl})\end{array}$ \\
\hline Canada (total) & 24650 & \\
\hline \multicolumn{3}{|l|}{ Sex } \\
\hline Female & 12514 & $48.6(48.6-48.6)$ \\
\hline Male & 12136 & $51.4(51.4-51.5)$ \\
\hline \multicolumn{3}{|l|}{ Grade } \\
\hline 9 & 7200 & $25.2(25.2-25.2)$ \\
\hline 10 & 6986 & $25.3(25.2-5.3)$ \\
\hline 11 & 6193 & $25.5(25.5-25.5)$ \\
\hline 12 & 4271 & $24.0(24.0-24.1)$ \\
\hline \multicolumn{3}{|l|}{ Ethnicity } \\
\hline White & 16970 & $60.9(52.3-69.4)$ \\
\hline Black & 859 & $4.7(3.0-6.3)$ \\
\hline Asian & 3597 & $22.7(14.6-30.9)$ \\
\hline Latin American & 434 & $2.3(1.2-3.3)$ \\
\hline Aboriginal & 1684 & $4.7(3.0-6.3)$ \\
\hline Other & 955 & $4.8(3.5-6.0)$ \\
\hline Missing values & 151 & \\
\hline \multicolumn{3}{|l|}{ Province } \\
\hline British Columbia & 3862 & $12.9(12.6-13.3)$ \\
\hline Alberta & 3957 & $10.8(10.5-11.1)$ \\
\hline Saskatchewan & 1895 & $3.2(3.1-3.3)$ \\
\hline Manitoba & 1863 & $4.0(3.9-4.1)$ \\
\hline Ontario & 3657 & $46.3(45.1-47.6)$ \\
\hline Quebec & 2608 & $15.9(15.5-16.3)$ \\
\hline Nova Scotia & 2778 & $2.7(2.6-2.7)$ \\
\hline Prince Edward Island & 1446 & $0.5(0.5-0.5)$ \\
\hline $\begin{array}{l}\text { Newfoundland and } \\
\text { Labrador }\end{array}$ & 2458 & $1.4(1.3-1.4)$ \\
\hline \multicolumn{3}{|l|}{$\begin{array}{l}\text { School socioeconomic } \\
\text { status }\end{array}$} \\
\hline Low median & 12066 & $44.9(28.6-61.1)$ \\
\hline High median & 12584 & $55.1(38.9-71.4)$ \\
\hline \multicolumn{3}{|l|}{ Urban } \\
\hline Yes & 15801 & $79.5(68.1-90.9)$ \\
\hline No & 8849 & $20.5(9.1-31.9)^{\star}$ \\
\hline \multicolumn{3}{|l|}{ Ever binge drink } \\
\hline Does not drink & 8316 & $38.8(34.4-43.2)$ \\
\hline Drinks but no binging & 4052 & $18.1(16.2-20.1)$ \\
\hline Binge drinks & 10955 & $43.1(39.6-46.5)$ \\
\hline Missing values & 1327 & \\
\hline \multicolumn{3}{|c|}{$\begin{array}{l}\text { Note: } \mathrm{Cl}=\text { confidence interval. } \\
\text { } \text { Presented as a proportion of complete data (i.e., does not include missing } \\
\text { values). }\end{array}$} \\
\hline
\end{tabular}


Relative to grade 11 students, grade 12 students had higher odds of ever driving after drinking (OR 1.642, 99\% CI 1.1152.417) or using marijuana (OR 1.584, 99\% CI 1.072-2.339). Drinking and driving did not vary by ethnicity. Relative to those identifying as white, students identifying as Aboriginal had higher odds of driving after using marijuana (OR 2.005, 99\% CI $1.140-3.526)$ and of being a passenger of a driver who had used marijuana within the previous 2 hours (OR 1.938, 99\% CI 1.364-2.755). Students who reported binge drinking had higher odds of engaging in each risky driving and passenger behaviour.

\begin{tabular}{|c|c|c|c|c|}
\hline \multirow[b]{2}{*}{ Variable } & \multicolumn{2}{|c|}{ In last $30 \mathrm{~d}$} & \multicolumn{2}{|c|}{ Ever } \\
\hline & $\begin{array}{l}\text { Weighted prevalence } \\
(95 \% \mathrm{Cl}), \%\end{array}$ & Odds ratio $(99 \% \mathrm{Cl})$ & $\begin{array}{c}\text { Weighted prevalence } \\
(95 \% \mathrm{CI}), \%\end{array}$ & Odds ratio $(99 \% \mathrm{Cl})$ \\
\hline Canada (total) & $3.5(2.8-4.2)$ & & $9.1(7.9-10.3)$ & \\
\hline \multicolumn{5}{|l|}{ Sex } \\
\hline Female & $2.7(1.8-3.5)$ & $0.516(0.264-1.011)$ & $6.8(5.3-8.2)$ & $0.477(0.290-0.786)$ \\
\hline Male & $4.3(3.2-5.4)$ & Reference & $11.4(9.4-13.4)$ & Reference \\
\hline \multicolumn{5}{|l|}{ Grade } \\
\hline 11 & $2.9(2.2-3.6)$ & Reference & $7.1(6.1-8.1)$ & Reference \\
\hline 12 & $4.2(3.2-5.1)$ & $1.406(0.883-2.240)$ & $11.3(9.2-13.4)$ & $1.642(1.115-2.417)$ \\
\hline \multicolumn{5}{|l|}{ Ethnicity } \\
\hline White & $3.7(2.7-4.6)$ & Reference & $10.4(8.5-12.3)$ & Reference \\
\hline Black & $\dagger$ & $2.645(0.730-9.584)$ & 7.1(2.6-11.5)‡ & $1.096(0.409-2.940)$ \\
\hline Asian & $1.6(0.7-2.5) \ddagger$ & $1.000(0.417-2.399)$ & $4.4(2.8-5.9) \ddagger$ & $0.850(0.440-1.643)$ \\
\hline Latin American & $\dagger$ & $5.238(0.278-98.553)$ & $16.7(6.5-27.0) \ddagger$ & $2.658(0.405-17.433)$ \\
\hline Aboriginal & $5.8(2.6-9.1) \ddagger$ & $1.441(0.518-4.007)$ & 14.7 (9.5-19.9)‡ & $1.031(0.529-2.008)$ \\
\hline Other & $\dagger$ & $2.433(0.743-7.968)$ & 10.3(4.7-15.3)‡ & $1.694(0.549-5.234)$ \\
\hline \multicolumn{5}{|l|}{ Province } \\
\hline British Columbia & $4.5(3.1-6.0)$ & $1.583(0.681-3.677)$ & $12.4(9.4-15.4)$ & $2.065(1.125-3.791)$ \\
\hline Alberta & $3.4(2.2-4.5) \ddagger$ & $1.339(0.558-3.214)$ & $9.5(7.2-11.8)$ & 1.699 (1.033-2.795) \\
\hline Saskatchewan & $9.8(4.9-14.8) \ddagger$ & $3.679(1.472-9.194)$ & $18.5(11.4-25.6) \ddagger$ & $2.926(1.510-5.670)$ \\
\hline Manitoba & $2.4(1.5-3.4) \ddagger$ & $0.630(0.254-1.564)$ & 11.1(5.8-16.4)‡ & $1.474(0.625-3.477)$ \\
\hline Ontario & $2.3(1.5-3.3) \ddagger$ & Reference & $6.3(4.4-8.2)$ & Reference \\
\hline Quebec & $\dagger$ & $1.007(0.367-2.764)$ & $9.0(7.0-11.0)$ & $1.213(0.656-2.243)$ \\
\hline Nova Scotia & $4.0(2.3-5.6) \ddagger$ & $1.109(0.433-2.839)$ & $10.0(7.9-12.1)$ & $1.299(0.796-2.120)$ \\
\hline Prince Edward Island & $3.8(2.7-4.9)$ & $1.174(0.562-2.458)$ & $11.7(8.3-15.0)$ & $1.462(0.799-2.674)$ \\
\hline Newfoundland and Labrador & $5.5(4.4-6.5)$ & $1.716(0.827-3.563)$ & 13.9(9.2-18.6)‡ & $1.828(1.042-3.208)$ \\
\hline \multicolumn{5}{|l|}{ School socioeconomic status } \\
\hline Low median & $3.6(2.6-4.6)$ & Reference & $10.5(8.0-13.0)$ & Reference \\
\hline High median & $3.5(2.5-4.4)$ & $1.219(0.605-2.457)$ & $8.0(6.7-9.4)$ & $0.889(0.518-1.526)$ \\
\hline \multicolumn{5}{|l|}{ Urban } \\
\hline Yes & $2.6(1.9-3.3)$ & Reference & $7.2(5.9-8.5)$ & Reference \\
\hline No & $7.6(5.9-9.4)$ & $2.326(1.312-4.125)$ & $17.6(13.7-21.4)$ & $1.712(1.163-2.520)$ \\
\hline \multicolumn{5}{|l|}{ Drinking behaviour } \\
\hline Does not drink & $\dagger$ & Reference & $\dagger$ & Reference \\
\hline Drinks but no binging & $0.7(0.4-1.0) \ddagger$ & $1.113(0.146-8.470)$ & $2.9(1.6-4.2) \ddagger$ & $2.391(0.527-10.839)$ \\
\hline Binge drinks & $5.9(4.9-7.0)$ & 8.559 (1.188-61.655) & $15.1(13.0-17.1)$ & 12.725 (3.284-49.308) \\
\hline
\end{tabular}

Note: $\mathrm{Cl}=$ confidence interval.

*All logistic regressions were conducted with the use of a complete-case methods approach, so the results presented here are among all cases with complete data. †High sampling variability/insufficient sample size; data suppressed.

†Moderate sampling variability; interpret with caution. 


\section{OPEN}

Research

No differences by school region socioeconomic status were observed. Relative to urban students, students from rural schools had higher odds of driving after drinking (OR 1.712, 99\% CI 1.163-2.520) and of ever riding with a driver who had drunk in the previous hour (OR 1.394, 99\% CI 1.128-1.723).

\section{Interpretation}

In Canada in 2014-2015, 9\% of grade 11-12 students reported ever driving within an hour of drinking alcohol or within 2 hours of using marijuana. Almost half (48\%) of grade 11-12

Table 3: Weighted prevalence and logistic regression analysis* of variables related to the odds of driving within 2 hours of using marijuana among 10411 grade 11-12 students

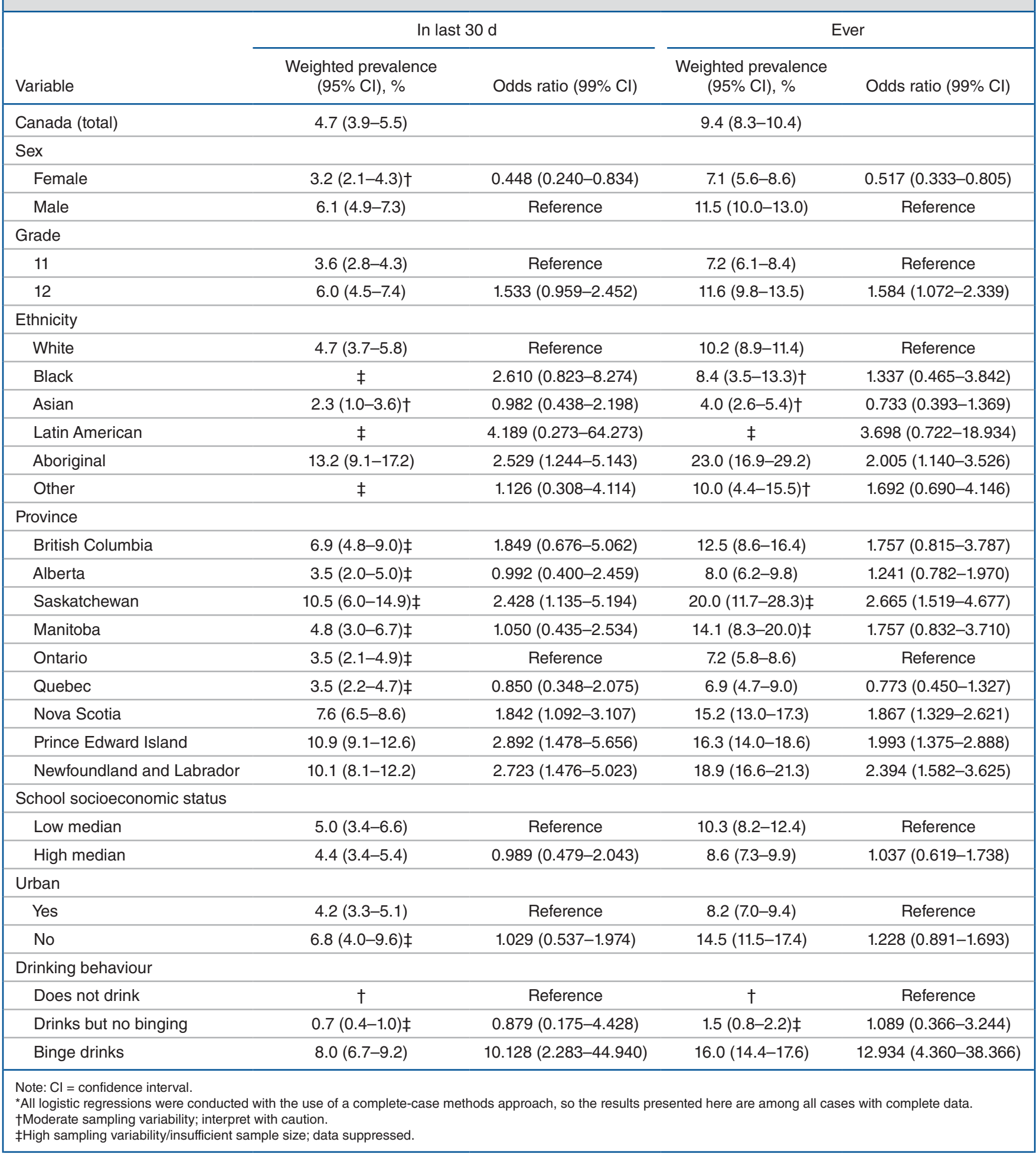


students reported having ever engaged in at least 1 risky driving or passenger behaviour. Significant differences in unsafe driving and passenger behaviours existed by individual charac- teristics such as sex, grade and binge drinking behaviours, but fewer differences in unsafe driving or passenger behaviours existed by area-level factors.

\begin{tabular}{|c|c|c|c|c|}
\hline \multirow[b]{2}{*}{ Variable } & \multicolumn{2}{|c|}{ In last $30 \mathrm{~d}$} & \multicolumn{2}{|c|}{ Ever } \\
\hline & $\begin{array}{c}\text { Weighted prevalence } \\
(95 \% \mathrm{Cl}), \%\end{array}$ & Odds ratio $(99 \% \mathrm{Cl})$ & $\begin{array}{l}\text { Weighted prevalence } \\
(95 \% \mathrm{Cl}), \%\end{array}$ & Odds ratio $(99 \% \mathrm{Cl})$ \\
\hline Canada (total) & $11.0(10.2-11.9)$ & & $34.6(32.4-36.9)$ & \\
\hline \multicolumn{5}{|l|}{ Sex } \\
\hline Female & $12.2(10.9-13.6)$ & $1.233(0.990-1.536)$ & $38.2(35.5-41.0)$ & $1.337(1.159-1.543)$ \\
\hline Male & $9.9(8.9-10.9)$ & Reference & 31.2 (29.1-33.3) & Reference \\
\hline \multicolumn{5}{|l|}{ Grade } \\
\hline 9 & $9.1(7.6-10.6)$ & Reference & $29.8(27.1-32.5)$ & Reference \\
\hline 10 & $11.1(9.9-12.2)$ & $0.952(0.698-1.301)$ & 34.5 (31.9-37.2) & $1.006(0.847-1.196)$ \\
\hline 11 & $12.4(11.1-13.7)$ & $0.995(0.737-1.344)$ & $36.9(34.7-39.1)$ & $1.045(0.869-1.257)$ \\
\hline 12 & $11.6(9.7-13.5)$ & $0.879(0.652-1.186)$ & $37.3(32.9-41.7)$ & $1.008(0.809-1.255)$ \\
\hline \multicolumn{5}{|l|}{ Ethnicity } \\
\hline White & $13.4(12.2-14.6)$ & Reference & $39.6(36.8-42.5)$ & Reference \\
\hline Black & $7.9(4.3-11.5) \dagger$ & $0.806(0.428-1.519)$ & $24.4(16.6-32.2)$ & $0.691(0.441-1.084)$ \\
\hline Asian & $5.5(4.6-6.5)$ & $0.682(0.492-0.946)$ & $22.4(20.5-24.3)$ & $0.798(0.626-1.017)$ \\
\hline Latin American & $7.7(4.8-10.7) \dagger$ & $0.604(0.342-1.066)$ & $39.7(32.4-47.0)$ & $1.112(0.697-1.774)$ \\
\hline Aboriginal & $12.4(9.8-15.0)$ & $0.860(0.564-1.312)$ & $42.7(37.6-47.8)$ & $1.014(0.740-1.389)$ \\
\hline Other & $11.0(8.1-13.9)$ & $1.051(0.662-1.667)$ & 30.1 (22.3-37.9) & $0.874(0.568-1.344)$ \\
\hline \multicolumn{5}{|l|}{ Province } \\
\hline British Columbia & $11.4(8.8-13.9)$ & $1.237(0.860-1.780)$ & $34.9(28.7-41.1)$ & $1.059(0.773-1.452)$ \\
\hline Alberta & $9.9(7.6-12.1)$ & $1.200(0.808-1.781)$ & $31.3(28.7-33.8)$ & $1.009(0.740-1.376)$ \\
\hline Saskatchewan & $14.7(11.3-18.1)$ & $1.444(1.029-2.026)$ & 38.7 (29.1-48.2) & $1.070(0.702-1.630)$ \\
\hline Manitoba & $9.1(7.0-11.1)$ & $0.888(0.578-1.366)$ & $30.5(24.4-36.6)$ & $0.790(0.516-1.210)$ \\
\hline Ontario & $9.1(7.8-10.3)$ & Reference & $30.9(26.7-35.1)$ & Reference \\
\hline Quebec & $15.4(12.4-18.5)$ & $1.309(0.881-1.946)$ & $43.8(40.0-47.5)$ & $1.204(0.855-1.694)$ \\
\hline Nova Scotia & $9.2(7.8-10.7)$ & $0.807(0.615-1.060)$ & $30.6(27.8-33.3)$ & $0.799(0.588-1.087)$ \\
\hline Prince Edward Island & $10.6(9.0-12.2)$ & $0.939(0.646-1.365)$ & $34.8(31.9-37.8)$ & $0.895(0.648-1.236)$ \\
\hline Newfoundland and Labrador & $10.0(8.3-11.7)$ & $0.864(0.684-1.090)$ & $31.4(26.2-36.7)$ & $0.789(0.579-1.076)$ \\
\hline \multicolumn{5}{|l|}{ School socioeconomic status } \\
\hline Low median & $12.2(10.9-13.6)$ & Reference & $40.0(37.0-43.0)$ & Reference \\
\hline High median & $10.1(8.8-11.4)$ & $0.997(0.807-1.231)$ & $30.3(27.1-33.4)$ & $0.789(0.620-1.004)$ \\
\hline \multicolumn{5}{|l|}{ Urban } \\
\hline Yes & $9.6(8.6-10.7)$ & Reference & $30.7(28.3-33.3)$ & Reference \\
\hline No & $16.4(14.1-18.6)$ & $1.094(0.841-1.425)$ & $49.5(46.2-52.8)$ & $1.394(1.128-1.723)$ \\
\hline \multicolumn{5}{|l|}{ Drinking behaviour } \\
\hline Does not drink & $3.5(2.8-4.1)$ & Reference & $17.0(14.8-19.2)$ & Reference \\
\hline Drinks but no binging & $8.5(6.8-9.5)$ & $2.259(1.432-3.561)$ & $32.9(29.1-36.8)$ & $2.072(1.600-2.685)$ \\
\hline Binge drinks & $18.5(17.1-20.0)$ & $5.425(3.681-7.995)$ & $49.8(47.1-52.6)$ & $3.893(3.138-4.829)$ \\
\hline
\end{tabular}




\section{OPEN}

Research

Almost 1 in 10 grade $11-12$ students reported ever driving after using alcohol or marijuana, which is comparable to the 2010 finding that $10 \%$ of Canadian 11 - to 15 -year-olds reported drinking and driving in the previous 30 days. ${ }^{11}$ However, it is higher than the rates found for Ontario, where $5 \%$ of grade 10-12 students with a driver's licence reported driv-

\begin{tabular}{|c|c|c|c|c|}
\hline \multirow[b]{2}{*}{ Variable } & \multicolumn{2}{|c|}{ In last $30 \mathrm{~d}$} & \multicolumn{2}{|c|}{ Ever } \\
\hline & $\begin{array}{l}\text { Weighted prevalence } \\
(95 \% \mathrm{Cl}), \%\end{array}$ & Odds ratio $(99 \% \mathrm{Cl})$ & $\begin{array}{c}\text { Weighted prevalence } \\
(95 \% \mathrm{Cl}), \%\end{array}$ & Odds ratio $(99 \% \mathrm{Cl})$ \\
\hline Canada (total) & $9.0(7.9-10.1)$ & & $19.8(18.0-21.6)$ & \\
\hline \multicolumn{5}{|l|}{ Sex } \\
\hline Female & $8.9(7.7-10.2)$ & $0.892(0.627-1.269)$ & $19.9(18.0-21.8)$ & $0.909(0.705-1.171)$ \\
\hline Male & $9.1(7.6-10.7)$ & Reference & $19.6(17.4-21.8)$ & Reference \\
\hline \multicolumn{5}{|l|}{ Grade } \\
\hline 9 & $4.0(2.9-5.0)$ & Reference & $9.1(6.9-11.3)$ & Reference \\
\hline 10 & $6.4(5.3-7.5)$ & $1.325(0.946-1.856)$ & $15.5(13.0-18.0)$ & $1.338(0.942-1.900)$ \\
\hline 11 & $10.6(9.2-11.9)$ & $1.872(1.196-2.929)$ & $22.5(20.5-24.4)$ & $1.893(1.310-2.735)$ \\
\hline 12 & $15.4(11.8-19.1)$ & $2.546(1.452-4.463)$ & $32.4(27.4-37.4)$ & $2.947(1.873-4.638)$ \\
\hline \multicolumn{5}{|l|}{ Ethnicity } \\
\hline White & $10.3(8.6-11.9)$ & Reference & $22.9(20.0-25.8)$ & Reference \\
\hline Black & $8.2(4.4-12.1) \dagger$ & $1.278(0.605-2.702)$ & 17.0 (11.7-22.2) & $1.097(0.599-2.011)$ \\
\hline Asian & $4.3(3.1-5.5)$ & $0.730(0.499-1.069)$ & $8.3(7.0-9.5)$ & $0.540(0.338-0.863)$ \\
\hline Latin American & $6.6(3.5-9.8) \dagger$ & $0.817(0.336-1.987)$ & $23.9(17.5-30.3)$ & $1.397(0.625-3.124)$ \\
\hline Aboriginal & $20.1(16.5-23.7)$ & 2.068 (1.453-2.942) & $38.8(33.0-44.6)$ & 1.938 (1.364-2.755) \\
\hline Other & $7.3(4.1-10.4) \dagger$ & $0.986(0.441-2.206)$ & $17.5(11.6-23.5) \dagger$ & $1.055(0.518-2.150)$ \\
\hline \multicolumn{5}{|l|}{ Province } \\
\hline British Columbia & $12.8(9.7-15.9)$ & $1.807(1.054-3.098)$ & $24.9(19.6-30.3)$ & $1.722(1.119-2.650)$ \\
\hline Alberta & $8.1(6.6-9.6)$ & $1.204(0.760-1.907)$ & 18.5 (16.9-20.1) & $1.336(0.946-1.885)$ \\
\hline Saskatchewan & $14.3(8.6-20.0) \dagger$ & $1.452(0.876-2.407)$ & $27.1(15.9-38.4) \dagger$ & $1.319(0.755-2.305)$ \\
\hline Manitoba & $10.0(7.2-12.9)$ & $1.015(0.587-1.755)$ & 22.5 (16.7-28.2) & $1.139(0.644-2.013)$ \\
\hline Ontario & $7.8(5.7-9.8)$ & Reference & $17.2(14.2-20.1)$ & Reference \\
\hline Quebec & $6.7(4.5-9.0) \dagger$ & $0.824(0.503-1.351)$ & $17.9(12.5-23.4) \dagger$ & $0.942(0.546-1.625)$ \\
\hline Nova Scotia & $13.8(11.9-15.7)$ & $1.612(1.008-2.576)$ & $27.8(25.4-30.3)$ & $1.706(1.142-2.548)$ \\
\hline Prince Edward Island & $15.3(13.6-17.1)$ & $1.863(1.192-2.912)$ & $29.2(26.9-31.6)$ & $1.743(1.181-2.571)$ \\
\hline Newfoundland and Labrador & $15.9(12.6-19.3)$ & $1.945(1.104-3.427)$ & $31.3(28.1-34.5)$ & $1.942(1.265-2.980)$ \\
\hline \multicolumn{5}{|l|}{ School socioeconomic status } \\
\hline Low median & $10.1(8.0-12.2)$ & Reference & $22.9(19.2-26.6)$ & Reference \\
\hline High median & $8.2(6.7-9.6)$ & $1.038(0.561-1.918)$ & $17.2(14.9-19.5)$ & $0.948(0.611-1.472)$ \\
\hline \multicolumn{5}{|l|}{ Urban } \\
\hline Yes & $8.5(7.2-9.8)$ & Reference & $18.2(16.1-20.4)$ & Reference \\
\hline No & $11.1(8.7-13.5)$ & $0.854(0.607-1.202)$ & $25.5(21.9-29.2)$ & $0.888(0.635-1.244)$ \\
\hline \multicolumn{5}{|l|}{ Drinking behaviour } \\
\hline Does not drink & $1.2(0.8-1.7) \dagger$ & Reference & $3.2(2.6-3.8)$ & Reference \\
\hline Drinks but no binging & $3.3(2.5-4.0)$ & $2.320(1.451-3.710)$ & $9.4(8.1-10.7)$ & $2.608(1.945-3.497)$ \\
\hline Binge drinks & $18.1(16.1-20.1)$ & 14.173 (8.335-24.099) & $37.5(34.5-40.5)$ & $13.693(10.300-18.203)$ \\
\hline
\end{tabular}

Note: $\mathrm{Cl}=$ confidence interval.

${ }^{*}$ All logistic regressions were conducted with the use of a complete-case methods approach, so the results presented here are among all cases with complete data. †Moderate sampling variability; interpret with caution. 
ing after drinking and 10\% reported driving after cannabis use. $^{22}$ In the current survey, just over one-third (35\%) of grade $9-12$ students reported being passengers of a driver who had drunk in the previous hour, and $20 \%$ reported riding with a driver who had used marijuana within the previous 2 hours. These rates are higher than those found in a 2008 survey: $14.6 \%$ of 15 - to 17 -year-olds rode as a passenger of a driver who had drunk, and $19.3 \%$ rode as a passenger of a driver who had used marijuana. ${ }^{10}$ Our estimates are also higher than the 2010 finding that $21 \%$ of 11 - to 15 -year-olds reported riding with a driver who had been using alcohol or marijuana or other illegal drugs in the previous 30 days. ${ }^{11}$ These differences are likely a function of survey methods, populations or survey instruments.

We found that, while boys had higher odds of driving after drinking or using marijuana, girls had higher odds of ever riding with drivers who had drunk. Males typically have higher odds of driving while impaired. ${ }^{11,13,24}$ Findings around the sexrelated nature of risky passenger behaviours are inconsistent: studies have shown no sex differences, ${ }^{24}$ that males are at higher risk ${ }^{10,11,13}$ and that females are at higher risk. ${ }^{25}$ Consistent with past research, in the current study, older adolescents $^{11,13,25}$ and those who reported binge drinking ${ }^{10,24,25}$ had higher odds of driving after drinking and riding with potentially impaired drivers. Few differences existed by schoolregion socioeconomic status. Compared with urban students, rural students had higher odds of reporting alcohol-related risky driving and passenger behaviours, consistent with previous research. ${ }^{11,13,25}$ Although there were few differences in alcohol-related risky behaviours, students from the 3 East Coast provinces for which generalizable data were available (Nova Scotia, Prince Edward Island and Newfoundland and Labrador) had higher odds of risky marijuana-related behaviours relative to Ontario students. Therefore, irrespective of the forthcoming federal approach to legalizing and regulating cannabis, it is within provincial jurisdiction to enact stricter provincial policies to reduce cannabis-impaired driving.

\section{Limitations and strengths}

Our study is subject to several limitations. First, outcome data were based on self-report rather than objective measures. Despite efforts to establish the validity and reliability of questionnaire items, ${ }^{26}$ some underreporting is likely. However, whereas objective data can measure the number of collisions related to impaired driving, no objective data exist on drinking or marijuana use before driving among youth. Furthermore, self-reported data are commonly used in similar studies. ${ }^{10,11,13,17}$ Second, survey items asked about driving within an hour of consuming 1 or more drinks of alcohol or within 2 hours of using marijuana. Although cognitive interviewing showed that these questions were easy for the target population to answer, they do not assess level of impairment, which depends on driver characteristics and on the amount of alcohol or marijuana consumed. Third, the survey did not include participants from Canada's territories, where the prevalence of impaired driving is high. ${ }^{27}$ However, the nonincluded populations represent only a small fraction of the Canadian population. Fourth, given the survey's focus on tobacco, alcohol and drug use and not driving behaviours per se, we were unable to determine whether students had driver's licences. ${ }^{25}$ Prevalence estimates of risky driving behaviours would likely have been higher had we restricted analyses to licensed youth. Despite these limitations, distinguishing between alcohol use and marijuana use before driving is a strength of the current study, particularly in light of the impending marijuana legalization in Canada. In addition, the comprehensive measures we used included risky driving of all types. The survey's national scope and provincially generalizable estimates are strengths.

\section{Conclusion}

A high proportion of Canadian youth reported engaging in risky driving and passenger behaviours. The impending legalization of marijuana necessitates further interventions to reduce impaired driving. Provincial policies can be implemented in the absence of federal legislation to achieve this end. Such policies may be particularly important for the Atlantic provinces, which had the highest prevalence of marijuanarelated risky driving and passenger behaviours. Future research should continue to monitor the prevalence of alcohol- and marijuana-related risky driving and passenger behaviours and should leverage surveillance data to conduct natural experiments on the impact of provincial policies as they are implemented.

\section{References}

1. Canadian motor vehicle traffic collision statistics. Ottawa: Transport Canada; 2014. Available: https://www.tc.gc.ca/media/documents/roadsafety/cmvtcs2014 eng.pdf (accessed 2016 Aug. 30).

2. Perreault S. Impaired driving in Canada, 2011. Ottawa: Statistics Canada; 2015 Available: www.statcan.gc.ca/pub/85-002-x/2013001/article/11739-eng.htm (accessed 2016 Aug. 30).

3. Percentage distribution for the 5 leading causes of death in Canada, 2011 [chart]. Ottawa: Statistics Canada; 2015. Available: www.statcan.gc.ca/pub/82 -625-x/2014001/article/11896/c-g/c-g01-eng.htm (accessed 2016 Apr. 22).

4. Ramage-Morin PL. Motor vehicle accident deaths 1979-2004. Health Rep 2008; 19:45-51.

5. Road safety in Canada. Ottawa: Transport Canada; 2014. Available: www. tc.gc.ca/eng/motorvehiclesafety/tp-tp15145-1201.htm\#s34 (accessed 2016 Aug. 30).

6. Brown SW, Vanlaar WGM, Robertson RD. Alcohol and drug crash problem in Canada: 2012 report. CCMTA Road Safety Research Report Series. Ottawa: Traffic Injury Research Foundation of Canada; 2015.

7. Summary of results: Canadian Student Tobacco, Alcohol and Drugs Survey 2014-15. Ottawa: Health Canada; 2016. Available: https://www.canada.ca/en/ health-canada/services/canadian-student-tobacco-alcohol-drugs-survey/2014 -2015-summary.html (accessed 2017 Mar. 5).

8. Mann RE, Stoduto G, Vingilis E, et al. Alcohol and driving factors in collision risk. Accid Anal Prev 2010;42:1538-44.

9. Williams AF. Alcohol-impaired driving and its consequences in the United States: the past 25 years. 7 Safety Res 2006;37:123-38.

10. Cartwright J, Asbridge M. Passengers' decisions to ride with a driver under the influence of either alcohol or cannabis. F Stud Alcohol Drugs 2011;72: 86-95.

11. Pickett $W$, Davison C, Torunian M, et al. Drinking, substance use and the operation of motor vehicles by young adolescents in Canada. PLoS One 2012; 7:e42807.

12. Beirness DJ. The characteristics of youth passengers of impaired drivers [technical report]. Ottawa: Canadian Centre on Substance Abuse; 2014. Available: www.ccsa.ca/Resource\%20Library/CCSA-Characteristics-Youth-Passengers -Impaired-Drivers-technical-report-2014-en.pdf (accessed 2017 Mar. 7).

13. Leadbeater BJ, Foran K, Grove-White A. How much can you drink before driving? The influence of riding with impaired adults and peers on the driving behaviors of urban and rural youth. Addiction 2008;103:629-37.

14. Adlaf EM, Begin P, Deguire AE. Canadian Addiction Survey (CAS): a national survey of Canadians' use of alcohol and other drugs - prevalence of use and related harms [detailed report]. Ottawa: Canadian Centre on Substance Abuse; 2005. 
15. Harris K. Senator Claude Carignan to table bill aimed at getting drugimpaired drivers off roads. CBC News 2016 Oct. 3. Available: www.cbc.ca/ news/politics/carignan-legislation-marijuana-impaired-driving-1.3786651 (accessed 2016 Oct. 4).

16. Canadian Press. Ontario introduces fines, licence suspensions for drugimpaired driving. Globe and Mail [Toronto]. Available: www.theglobeandmail. $\mathrm{com} /$ news/national/ontario-introduces-fines-licence-suspensions-for-drug -impaired-driving/article32104976/ (accessed 2017 Mar. 7).

17. Asbridge M, Hayden JA, Cartwright JL. Acute cannabis consumption and motor vehicle collision risk: systematic review of observational studies and meta-analysis. BM7 2012;344:e536.

18. Mura P, Kintz P, Ludes B, et al. Comparison of the prevalence of alcohol, cannabis and other drugs between 900 injured drivers and 900 control subjects: results of a French collaborative study. Forensic Sci Int 2003;133:79-85.

19. Laumon B, Gadegbeku B, Martin JL, et al.; SAM Group. Cannabis intoxication and fatal road crashes in France: population based case-control study [published erratum in BMF 2006;332:1298]. BMF 2005;331:1371.

20. Li MC, Brady JE, DiMaggio CJ, et al. Marijuana use and motor vehicle crashes. Epidemiol Rev 2012;34:65-72.

21. Hartman RL, Huestis MA. Cannabis effects on driving skills. Clin Chem 2013; 59:478-92.

22. Boak A, Hamilton HA, Adlaf EM, et al. Drug use among Ontario students, 1977-2015: OSDUHS highlights. CAMH Research Document Series no. 42. Toronto: Centre for Addiction and Mental Health; 2015.

23. Census metropolitan area (CMA) and census agglomeration (CA). Ottawa: Statistics Canada; 2015. Available: https://www12.statcan.gc.ca/census-recensement/ 2011/ref/dict/geo009-eng.cfm (accessed 2017 Mar. 20).

24. Li K, Simons-Morton BG, Hingson R. Impaired-driving prevalence among US high school students: associations with substance use and risky driving behaviors. Am 7 Public Health 2013;103:e71-7.

25. Poulin C, Boudreau B, Asbridge $M$. Adolescent passengers of drunk drivers: a multi-level exploration into the inequities of risk and safety. Addiction 2007; 102:51-61.

26. Elton-Marshall T, Leatherdale ST, Manske SR, et al. Research methods of the Youth Smoking Survey (YSS). Chronic Dis Inj Can 2011;32:47-54.

27. Canadians who reported they have, at least once in the preceding 12 months, driven after having two drinks in the preceding hour, by age and sex, selected provinces and territories, 2008 and 2010 [table]. Ottawa: Statistics Canada; 2015. Available: www.statcan.gc.ca/pub/85-002-x/2013001/article/11739/tbl/ tbl04-eng.htm (accessed 2016 Sept. 19).
Affiliations: School of Planning, Faculty of Environment (Minaker); Propel Centre for Population Health Impact (Minaker, Bonham), University of Waterloo, Waterloo, Ont.; Institute for Mental Health Policy Research (Elton-Marshall), Centre for Addiction and Mental Health, London, Ont.; Dalla Lana School of Public Health (Elton-Marshall), University of Toronto, Toronto, Ont.; School of Public Health and Health Systems (Leos-Toro, Hammond), University of Waterloo, Waterloo, Ont.; School of Public Health (Wild), University of Alberta, Edmonton, Alta.

Contributors: Leia Minaker conceived of and designed the study and drafted the manuscript. Aaron Bonham analyzed the data. Tara EltonMarshall, Cesar Leos-Toro, T. Cameron Wild and David Hammond contributed to writing the manuscript and provided substantive feedback on revisions. All of the authors were involved in interpreting the data, approved the final version to be published and agreed to act as guarantors of the work.

Funding: This project was supported by grant 2011-701019 from the Canadian Cancer Society, through the Propel Centre for Population Health Impact. Leia Minaker was supported by a Career Development Award from the Canadian Cancer Society (no. 704744). Additional support was provided by a Canadian Institutes of Health Research Applied Public Health Chair to David Hammond.

Acknowledgement: The authors thank Robert Solomon for providing excellent suggestions to improve the clarity of the manuscript.

Disclaimer: Data used for this research were taken from Health Canada's Canadian Student Tobacco, Alcohol and Drugs Survey (formerly Youth Smoking Survey), which was conducted for Health Canada by the Propel Centre for Population Health Impact at the University of Waterloo. Health Canada has not reviewed, approved or endorsed this research. Any views expressed or conclusions drawn herein do not necessarily represent those of Health Canada.

Supplemental information: For reviewer comments and the original submission of this manuscript, please see www.cmajopen.ca/content/5/2/ E386/suppl/DC1 